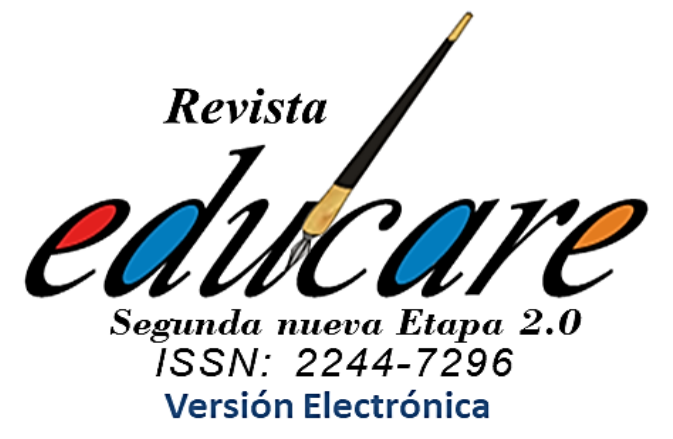

Volumen 24 № 1 Enero-Abril 2020

$(180-197)$

Martha Judith Pereira Valdez ORCID: https://orcid.org/0000-0002-9117-0800

Cristhian Fernando Velastegui ORCID: https://orcid.org/0000-0002-8837-9945

Bertha Susana Paz Viteri: ** ORCID: https://orcid.org/0000-0001-9240-1941

UNIVERSIDAD CENTRAL DEL ECUADOR ECUADOR

*Martha Judith Pereira Valdez: Magister en Cultura Física y entrenamiento Deportivo, docente en la Universidad Técnica de Machala, correo mpereira@utmachala.edu.ec

Cristhian Fernando Yancha Velastegui: Magister en Cultura Física y Entrenamiento Deportivo, Preparador físico de la asociación de árbitros profesionales de Tungurahua, correo aapft@yahoo.es

Betha Susana Paz Viteri: Magister en Cultura Física y Entrenamiento Deportivo, docente en la Universidad Nacional de Chimborazo, correo spaz@unach.edu.ec
REPÚBLICA BOLIVARIANA DE VENEZUELA UNIVERSIDAD PEDAGÓGICA EXPERIMENTAL LIBERTADOR INSTITUTO PEDAGÓGICO DE BARQUISIMETO "LUIS BELTRÁN PRIETO FIGUEROA

SUBDIRECCIÓN DE INVESTIGACIÓN Y POSGRADO BARQUISIMETO ESTADO LARA

\section{PRÁCTICAS DEPORTIVAS Y ENFOQUES CURRICULARES: UNA PERSPECTIVA DESDE LA EDUCACIÓN FÍSICA ECUATORIANA}

\author{
SPORTS PRACTICES AND CURRICULAR \\ APPROACHES: A PERSPECTIVE FROM \\ ECUADORIAN PHYSICAL EDUCATION
}

\section{Recibido:}

06-11-2019

Aceptado:

12-02-2020 

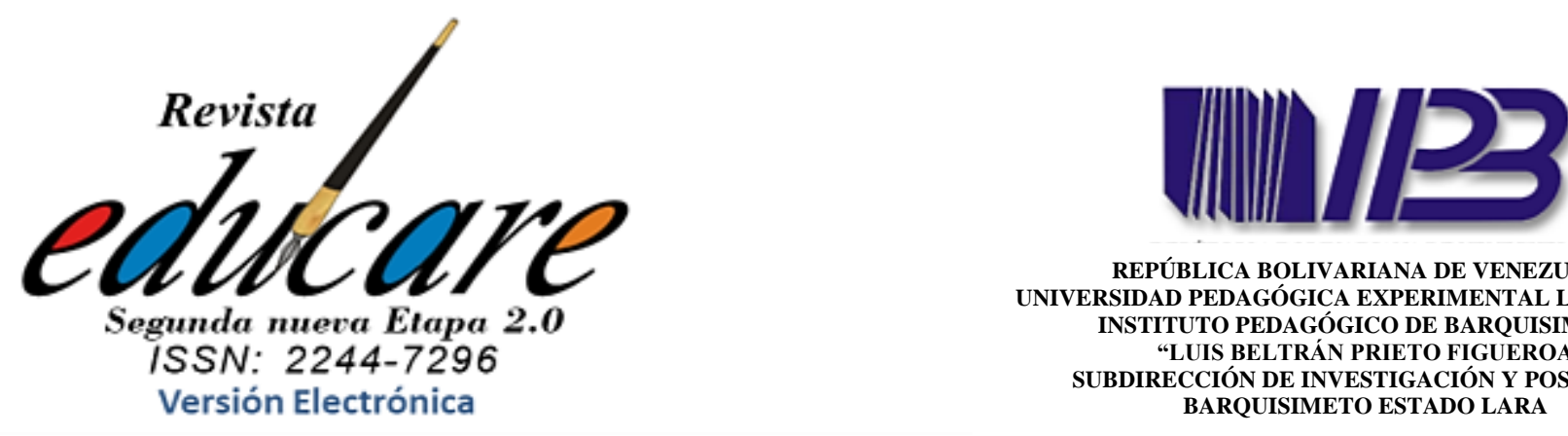

REPÚBLICA BOLIVARIANA DE VENEZUELA UNIVERSIDAD PEDAGÓGICA EXPERIMENTAL LIBERTADOR INSTITUTO PEDAGÓGICO DE BARQUISIMETO "LUIS BELTRÁN PRIETO FIGUEROA SUBDIRECCIÓN DE INVESTIGACIÓN Y POSGRADO BARQUISIMETO ESTADO LARA

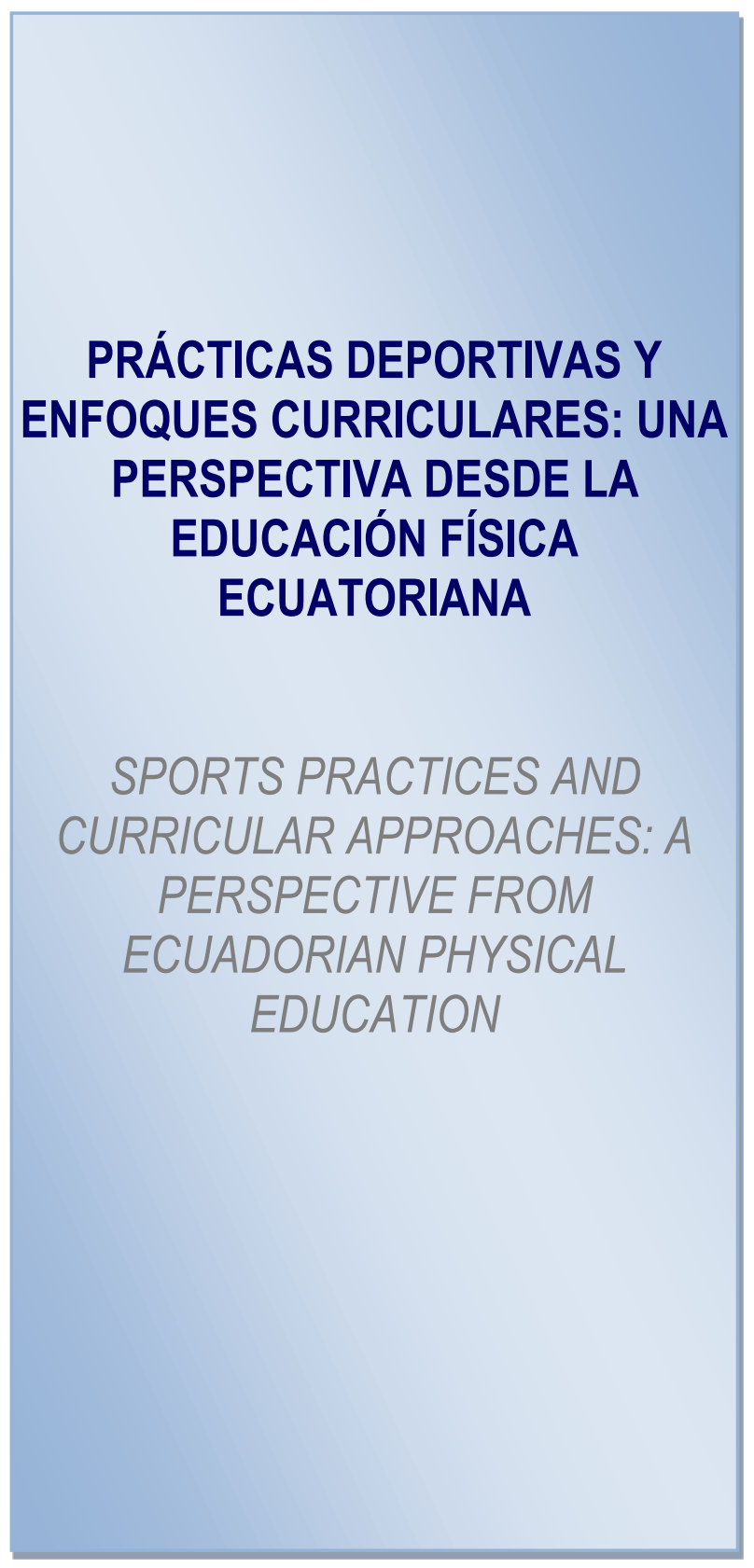

\section{Resumen}

El currículo de la Educación Física ecuatoriano desarrolla el bloque prácticas deportivas con enfoques inclusivo, lúdico y corporeidad. El estudio tuvo como objetivo reflexionar sobre el desarrollo del deporte y los enfoques curriculares en las clases de educación física; se utilizó el enfoque cualitativo interpretativo debido a la comprensión de un fenómeno educativo como una realidad múltiple y socialmente construida. La población estuvo conformada por nueve docentes de educación física pertenecientes a tres instituciones educativas, se aplicó una entrevista libre, con base a los hallazgos se obtuvieron dos criterios el entrenamiento deportivo en las clases de Educación Física y los enfoques curriculares en clases de Educación Física y con ellos se realizó una triangulación de datos espacial, obteniendo la reflexión de que las prácticas deportivas no debe ser direccionada hacia el entrenamiento deportivo sino a la formación integral del estudiante en lo motriz, cognitivo, afectivo y social.

Descriptores: educación física, deporte, práctica deportiva, currículo, enfoque

\section{Abstract}

The curriculum of Ecuadorian Physical Education develops the block of sports practices with inclusive, playful and corporeity approaches. The study aimed to reflect on the development of sport and curriculum approaches in physical education classes; the qualitative interpretative approach was used due to the understanding of an educational phenomenon as a multiple and socially constructed reality. The population consisted of nine physical education teachers belonging to three educational institutions, a free interview was applied, based on the findings, two criteria were obtained sports training in physical education classes and curricular approaches in physical education classes and with them a triangulation of spatial data was carried out, obtaining the reflection that sports practices should not be directed towards sports training but to the integral training of the student motor, cognitive, affective and social.

Keywords: physical education, sport, sports practice, curriculum, focus. 


\section{INTRODUCCIÓN}

La educación, con un significado amplio, pretende ser una forma de promover el desarrollo de las habilidades, destrezas y motivaciones del individuo para lograr su crecimiento personal y social. En este sentido, la Organización de las Naciones Unidas para la Ciencia y la Cultura, UNESCO (1998) enfatizó que la educación es necesaria para proporcionar a los niños, jóvenes $\mathrm{y}$ adultos los conocimientos y habilidades necesarias para tomar decisiones informadas en la mejora de la salud y el nivel de vida. Desde esta perspectiva, el propósito básico de la educación es elevar los niveles de desarrollo afectivo, cognitivo, axiológico, psicomotor, social, ecológico y, por lo tanto, en la sociedad en la que se desarrolla.

Las reformas curriculares en los países latinoamericanos han sufrido un constante cambio en los paradigmas educativos, sin embargo han servido para avanzar en la calidad educativa como lo expresa Román y Díez (2000):

Las Reformas Educativas Iberoamericanas actuales existe un importante desarrollo del paradigma cognitivo y sus teorías subyacentes, con conceptos tales como objetivos verticales por capacidades, objetivos transversales por valores, aprendizaje constructivo y significativo, aprender a aprender, evaluación por procesos ,... Pero estas reflexiones cuando llegan a la práctica se concretan en modelos conductistas, con diseños curriculares centrados en lo observable, medible y cuantificable, a partir de actividades orientadas al aprendizaje de contenidos como formas de saber o al aprendizaje de métodos como formas de hacer (p. 2)

De acuerdo con lo anterior, Posso, Barba, Marcillo, Acuña y Hernández (2020) los modelos educativos se transforman de acuerdo a las necesidades de la sociedad, en este sentido la Educación Física se encamina en este cambio, es decir al desarrollo del paradigma socio constructivista que va de la mano con los enfoques curriculares lúdico, inclusivo y de la corporeidad como lo señalan Posso, Barba, Castro, Nuñez y Marcillo (2019) al describir que las clases de Educación Física deben tener un ambiente de diversión y esparcimiento conjugado con la participación activa de todos los estudiantes, generando el reconocimiento corporal, en busca de masificar una cultura de actividad física.

Desde esta perspectiva, la Educación Física en cada nivel, debe estar orientada al logro de la autorrealización del estudiante, entendida esta como la orientación de la personalidad, hacia el desarrollo de las potencialidades del individuo que conlleve a su formación integral. En este marco de circunstancias, para la formación de pautas conductuales, morales, espirituales y 
transmisión de conocimientos, que permitan al hombre conocerse a sí mismo con intencionalidad constructiva y al entorno, como escenario de su desarrollo vital; en este sentido, es pertinente señalar el área de Educación Física, como uno de los componentes curriculares que conforman el pensum de estudio, como una unidad que hace posible caracterizar las acciones y expresiones sustantivas para lograr el desarrollo de su corporeidad, su parte social y emocional para ser aplicada durante toda su vida; en este mismo planteamiento Monzonís y Capllonch (2015) mencionan sus perspectivas sobre el desarrollo de la Educación Física:

Estas ideas siguen los planteamientos educativos que parten del desarrollo de las competencias básicas ya que se entiende que tienen que ver con la capacidad de los estudiantes de extrapolar lo que han aprendido para aplicar sus conocimientos en nuevas circunstancias, así como conectar su aprendizaje a lo largo de la vida con regularidad. Está conexión entre competencias y vivencias contribuirá al despliegue de una trayectoria personal exitosa y al buen funcionamiento de la sociedad, porque son importantes para las esferas de la vida del individuo y para la sociedad. (p. 259)

Cabe destacar, que según Flores, Prat y Soler (2015), el área de Educación Física comprende la enseñanza de la psicomotricidad, valorando las diferentes edades de los estudiantes, para favorecer el desarrollo físico de los niños, así como su equilibrio emocional, psíquico y salud en general, como un área específica. Por otra parte, contribuye a perfeccionar el desarrollo de las habilidades motrices básicas: caminar, correr, saltar, lanzar, atrapar, halar, empujar, transportar, escalar y reptar, así como a la internalización, por parte del estudiante, de las nociones básicas sobre la actividad física y su importancia para la salud; también la Educación Física comprende la enseñanza de la recreación ante lo cual Posso, Aponte, Zapata y Betancourt (2020) señalan que el juego es pate importante del que hacer educativo, empoderando a los estudiantes a trabajar con el enfoque lúdico en cada actividad que se realice, apoyando este enunciado Arufe (2020) menciona que:

El juego, la experimentación y la actividad práctica deben constituir el vehículo principal para la enseñanza de todos los contenidos. También el espacio, los materiales y el tiempo deben planificarse de forma correcta y especialmente crear espacios que generen aprendizaje. La propia arquitectura del aula es también una gran fuente educativa, dado que los espacios también educan, destacando el importante papel desarrollado por los rincones de aprendizaje donde se puedan trabajar diferentes contenidos y habilidades previamente planificados por el docente. (p. 590)

Por su parte, Rodríguez - Rodríguez, Curilem-Gatica, Escobar-Gómez y ValenzuelaEberhard (2016) agregan que la Educación Física además propicia el desarrollo deportivo en el 
ámbito escolar, mediante una preparación física del individuo, para dotar a su aparato locomotor de resistencia y fuerza, con lo que reduce al mínimo la frecuencia de problemas de salud o patologías causadas por los esfuerzos y por ende optimiza el estado psicológico del individuo. Desde esta perspectiva, el deporte se presenta como la actividad física, que si se pretende, puede asumir carácter competitivo, según sea el caso; pero sin dejar de considerar que su verdadero propósito es contribuir a mejorar la condición física e integral de quien lo practica.

Dentro de la disciplina deportivas es importante destacar la práctica del deporte de rendimiento, como proceso utilizado para optimizar el aprovechamiento de las capacidades corporales del individuo y desarrollar al máximo las habilidades y destrezas del mismo, mediante el entrenamiento y el uso de los recursos disponibles. De esta manera, adquiere su formación integral, las destrezas que le permitirán, en un futuro, alcanzar un alto nivel competitivo, Renzi (2009) menciona que la Educación Física es:

Es una disciplina pedagógica que busca intervenir intencional y sistemáticamente en la formación integral de los alumnos, a través de su incidencia específica en la constitución y desarrollo de su corporeidad y su motricidad, adecuando sus intervenciones a los diversos contextos socioculturales. Más allá de su denominación, es preciso abandonar la mirada positivista que aún limita a esta disciplina a una educación de lo físico, del organismo biológico o del comportamiento motor. En cambio, el sujeto pedagógico de la Educación Física es el ser humano en su unidad y globalidad, y su singularidad radica en que se lleva a cabo mediante la corporeidad y la motricidad del mismo. (p. 1)

En este sentido aunque se quiera dar una Educación Física deportiva, no se debe dejar de lado el contexto educativo, es decir manejar los procesos de enseñanza aprendizaje, por lo que el sentido de entrenamiento se elimina, para pasar a la enseñanza cooperativa y constructivista, afianzando los contenidos para la vida.

Desde la Educación Física, el deporte de rendimiento, como parte de la formación integral del estudiante; requiere no solo la preparación física del deportista para desarrollar su potencial, sino además su preparación biológica, psicológica, teórica, técnica y táctica, que se logra a través de un entrenamiento exhaustivo, con métodos específicos y sustentados en principios definidos, para que dicho estudiante comience a disciplinarse, establecer metas y objetivos, y busque siempre mejorar la actividad, por medio de una práctica continua, lo cual más adelante puede conllevarlo a la práctica del deporte de alto rendimiento. Cabe destacar que para Rodríguez-Rodríguez, Curilem-Gatica, Escobar-Gómez y Valenzuela-Eberhard (2016), el 
deporte de rendimiento como disciplina deportiva, ya sea tradicional o no tradicional, requiere desarrollarse con alta rigurosidad y desempeño en el área donde se especialice. Gómez, Padial, Gentil, Chacón y Zurita (2019) están de acuerdo con esta posición al acotar:

Un factor importante a tener en cuenta a la hora de motivar al alumnado en su participación en los contenidos relacionados con el deporte en la escuela, es el modelo de competición. Solo un $4 \%$ de la muestra indica como motivante la competición a la hora de realizar deporte. Esta motivación por ganar, está relacionada con el considerable porcentaje de abandono en la práctica deportiva de adolescentes, puesto que produce decepción al no satisfacer las expectativas de rendimiento.

Con estos planteamientos se puede decir que en la práctica deportiva en educación física se debe disminuir la motivación de la competencia, debido también al sentido de exclusión y de que es permisible del bullyng, por lo contrario se debe aumentar el motivante de la cooperación en la construcción de actividades con la transversalización de los tres enfoques del currículo ecuatoriano y la activación de las metodologías alineadas.

Dentro de la perspectiva pedagógica, la educación física constituye un área de aprendizaje específica, cuya misión educativa se cristaliza al proporcionar experiencias motoras variadas, divertidas y de participación activa de todos los estudiantes durante el tiempo de clase, tanto motoras como cognitivas, intelectual, emocional y social. Cargua, Posso, Cargua y Rodríguez (2019) mencionan que en todos los procesos de enseñanza de Educación Física los docentes deben ser innovadores y creativos en el diseño de las actividades y posteriormente planificar para que los estudiantes los puedan construir.

De acuerdo a los contenidos planteados por el Currículo Nacional de Educación Física del Ecuador, Posso (2018) menciona que el bloque curricular Prácticas Deportivas se desarrolla desde el subnivel media del nivel Educación General Básica, es decir a partir de los nueve años, debido a que se considera que a esa edad se ha terminado de consolidar las capacidades perceptivo motrices, pudiendo trabajar en lo técnico- táctico de los diferentes deportes. En este mismo sentido Posso, et al. (2020) mencionan que los bloques curriculares son:

Se clasifican en Prácticas lúdicas que abarcan destrezas con criterio de desempeño que desarrollan actividades relacionadas a los juegos en sus diferentes tipos y de acuerdo al contexto en que se desarrollan, Prácticas gimnásticas que abarcan destrezas con criterio de desempeño relacionadas al desarrollar actividades de carácter gimnástico y desarrollo de las capacidades físicas y coordinativas de acuerdo al desarrollo psicomotriz de los estudiantes, Prácticas corporales expresivo 
comunicativas que abarca destrezas con criterio de desempeño que desarrollan todas las actividades corporales que comunican y expresan, Prácticas deportivas que abarcan destrezas con criterio de desempeño que desarrollan todas las habilidades, técnicas tácticas y estrategias deportivas, de acuerdo a los intereses y necesidades de los estudiantes; además se manejas dos bloques transversales como la construcción de la identidad corporal y las relaciones de las prácticas corporales con las salud. (p. 135)

Se debe considerar que los docentes podrán contextualizar los contenidos curriculares, es decir si en la destreza con criterio de desempeño o contenido curricular se plantea el desarrollo técnico de prácticas deportivas, los docentes deberán de acuerdo a las necesidades e intereses de los estudiantes, a la infraestructura, a los recursos e inclusive al conocimiento del propio docente elegir el deporte a practicar. Según Bernáez et al. (2007), citado por Tinedo (2012), el deporte es concebido como:

...una actividad de trabajo físico caracterizado por el espíritu de competencia reglamentada, realizada por el placer mismo que la actividad promueve. El hombre entra en juego todas sus fuerzas, toda su inteligencia, todo su valor, toda su formación moral, en donde hay gasto energético, alteración del estado normal y funcional del hombre (p. 3).

La Educación Física es la asignatura base en la cual se puede identificar talentos deportivos cuya definición lo plantea Labarrere (1982), como “don innato o disposición natural situada por encima del nivel medio del hombre normal en un determinado ámbito y que no está determinado por una sola capacidad sino por la combinación de las capacidades que permiten desarrollar la actividad compleja"; Soto y Andújar, (2000) también menciona que talento deportivo es el "conjunto de facultades o aptitudes para una cosa; una aptitud natural o adquirida para hacer algo. Depende de la capacidad individual del sujeto pero también de una serie de aspectos externos e internos, como las condiciones sociales y afectivas"

También se puede de manera paralela crear en los niños una cultura de cuerpo y mente sana, que propicie su desarrollo integral; de esta manera, este proceso permite el desarrollo de talento deportivo, como lo plantea Medranda-Rojas, Castillejo-Olán, Pérez-Ramírez y AlonsoBetancourt. "El docente tiene que desplegar preparación psicológica y pedagógica para enfrentar la dirección de la Educación Física" esto permitirá identificarlos ocupando espacios deportivos adecuados, sino cualquier espacio educativo durante las clases de Educación Física, que promueven el desarrollo físico y mental de los estudiantes, con actividades deportivas caracterizadas por el apoyo técnico, orientado a obtener una actividad activa, saludable y vida 
integrada; Fernández (2005), plantea también que el talento deportivo debe desarrollar “niño, adolescente o joven muestra cualidades, condiciones que lo distinguen de otros, lo que expresa posibilidades de tener un resultado superior a los demás en una actividad deportiva determinada"

En este sentido para desarrollar excelentes condiciones físicas y destrezas deportivas en los estudiantes, el docente conoce sobre la técnica y táctica deportiva al enseñar su accionar no solo físico sino también psicológica; debe ser persuasivo, con la habilidad de inducir a los demás a creer o hacer las actividades desarrollando vinculando los tres enfoques curriculares; se debe considerar que los estudiantes tienen somatotipos casi similares los cuales no interfieren ni influyen directamente en la práctica activa deportiva ; aportando estas ideas Posso, Otáñez, Guerrero, Betancourt, Noroña y Manangón (2020) mencionan un estudio realizado en niños:

Los nadadores (femenino y masculino) de los centros deportivos de QuitoEcuador, poseen un somatotipomeso endomórfico. Desde el punto de vista inferencial, se concluye que ambos grupos, tanto nadadores como voleibolistas, presentan un alto índice de correlación entre las variables somatotípicas estudiadas, ubicándose en el nivel de alta a muy alta, en donde la única diferencia significativa, se observó en la variable de peso. Esto permite evidenciar, que tanto nadadores como voleibolistas presentan características somatotípicas similares. (p. 11)

Conociendo esto el docente también debe ser dinámico, para emprender de manera activa, acciones de acuerdo al contexto; participativo, que intervenga en tareas y trabajo en equipo. Además, debe reunir conocimientos teóricos y metodológicos de enseñanza, cabe señalar que el resultado deportivo no va a generar la evaluación sino el aprendizaje de la técnica o táctica, que se podrá emular para toda su vida.

En las clases de Educación Física todo ejercicio que sirva para desarrollar la actividad deportiva debe iniciar obligatoriamente con el calentamiento direccionado a acondicionar el organismo a la práctica de las mismas con trabajo articular, aeróbico, estiramiento y de activación; en este caso no se debe dejar de lado los elementos de una clase educativa que será conocimiento previo, planteamiento del tema y objetivo de la clase.

Por último se debe diferenciar el entrenamiento deportivo y la práctica deportiva en las clases de Educación Física; en el deporte de rendimiento el objetivo fundamental es el aumento de nivel competitivo en el deportista, es preciso además lograr el incremento de todos los indicadores de la preparación, que según Platonov (1987), son: el factor psicológico, teórico, 
físico, técnico y táctico, los cuales deben formar una estrecha relación con el proceso deportivo planificado; pero la práctica deportiva en Educación Física es el educativa de formación para ser autónomo del ejercicio físico y como despertar la iniciativa y motivación al deporte, en tal virtud el docente direccionará a los estudiantes a entrenamientos extracurriculares que lo perfeccionarán en el deporte escogido.

\section{METODOLOGÍA DE LA INVESTIGACIÓN}

Esta investigación está sustentada con el enfoque cualitativo interpretativo que según Maykut y Morehouse (1994) mencionan que este enfoque debido a que se trató de la comprensión y descripción de lo entendido reconstruyendo la información en un contexto reconocible, también González-Martínez (1993) menciona que "la metodología cualitativa las variables no están predefinidas, más bien se van construyendo como resultado del tratamiento inductivo que se da a los datos; las variables surgen de lo que los sujetos estudiados, desde su propia perspectiva" es decir en este fenómeno educativo que es la reflexión de la práctica deportiva y la inclusión de los enfoques curriculares en las clases de Educación Física se hace necesario la interpretación de los datos recopilados para obtener una narrativa descriptiva de este fenómeno.

En este estudio se aplicó la muestra censal según Hurtado y Toro (2001) dicen que "en las poblaciones pequeñas o finitas no se selecciona muestra alguna para no afectar la valides de los resultados". (p.77), es por eso que la población es la misma muestra, por lo que estuvo conformada por nueve docentes de educación física pertenecientes a tres instituciones educativas.

El instrumento que se aplicó fue una entrevista de carácter libre, Alonso (2007) menciona que este instrumento de recolección de datos es una conversación acordada entre dos personas una entrevista y la otra informa, y tiene la finalidad de producir datos relevantes para una investigación, Díaz-Bravo, Torruco-García, Martínez-Hernández y Varela-Ruiz (2013) mencionan que la entrevista es:

Muy ventajosa principalmente en los estudios descriptivos y en las fases de exploración, así como para diseñar instrumentos de recolección de datos, la entrevista en la investigación cualitativa, independientemente del modelo que se decida emplear, se caracteriza por los siguientes elementos: tiene como propósito obtener información en relación con un tema determinado; se busca que la información recabada sea lo más precisa posible; se pretende conseguir los 
significados que los informantes atribuyen a los temas en cuestión; el entrevistador debe mantener una actitud activa durante el desarrollo de la entrevista, en la que la interpretación sea continua con la finalidad de obtener una compresión profunda del discurso del entrevistado. Con frecuencia la entrevista se complementa con otras técnicas de acuerdo a la naturaleza específica de la investigación. (p. 162)

En esta entrevista se planteó diez preguntas las cuales ingresaron a un proceso de validación por siete expertos, que fueron seleccionados de acuerdo a los parámetros establecidos por Skjong y Wentworht (2000) experiencia docente, reconocimiento académico y disponibilidad de tiempo para la validación. Se realizó una circulación debido a que no existieron observaciones y sugerencias de exclusión, inclusión, corrección y contextualización del banco de preguntas establecidas para esta investigación.

\section{Análisis de Hallazgos}

La entrevista contribuyó con información esencial para esta investigación, debido a que se pudo averiguar si en las clases de Educación Física se desarrolla el deporte o las prácticas deportivas con la tranversalización de los tres enfoques curriculares. Se realizó las entrevistas enfocadas a todos los docentes de Educación Física de tres instituciones educativas particulares de la ciudad de Quito, siguiendo los lineamientos de la entrevista se informó los objetivos de la misma cumpliendo con lo establecido con la American Psychological Association (2010) en cuanto a la normativa ética.

Los resultados obtenidos de las entrevistas se agruparon por instituciones educativas, debido a que en cada una existe libertad curricular en el contexto de un currículo de Educación Física abierto y flexible. Además estos resultados se dividieron en dos criterios y categorías, a fin de interpretar los hallazgos encontrados. 
Tabla 1

Resultados de las entrevistas

\begin{tabular}{|c|c|c|c|c|c|}
\hline Criterios & Categorías & $\begin{array}{l}\text { Instituci } \\
\text { ón } 1\end{array}$ & $\begin{array}{l}\text { Instituc } \\
\text { ión } 2\end{array}$ & $\begin{array}{l}\text { Instituc } \\
\text { ión } 3\end{array}$ & $\begin{array}{l}\text { No. } \\
\text { Docen } \\
\text { tes }\end{array}$ \\
\hline \multirow[t]{9}{*}{$\begin{array}{l}\text { Entrenamiento } \\
\text { Deportivo en las clases } \\
\text { de Educación Fisica }\end{array}$} & $\begin{array}{l}\text { Entrenamiento } \\
\text { deportivo propio de } \\
\text { clases de Educación } \\
\text { Física. }\end{array}$ & $\mathbf{1}$ & - & 2 & 3 \\
\hline & $\begin{array}{l}\text { Entrenamiento } \\
\text { deportivo excluyente. }\end{array}$ & $\mathbf{1}$ & 3 & $\mathbf{1}$ & 5 \\
\hline & $\begin{array}{l}\text { Perfección de las } \\
\text { técnicas y tácticas. }\end{array}$ & $\mathbf{1}$ & - & 2 & 3 \\
\hline & $\begin{array}{l}\text { Potencialización de las } \\
\text { Capacidades Fisicas. }\end{array}$ & 2 & - & $\mathbf{1}$ & 3 \\
\hline & $\begin{array}{l}\text { Detección de talentos } \\
\text { deportivos. }\end{array}$ & 2 & - & 2 & 4 \\
\hline & Formación deportiva. & 2 & 1 & 1 & 4 \\
\hline & $\begin{array}{l}\text { Especialización } \\
\text { deportiva. }\end{array}$ & 2 & - & 2 & 4 \\
\hline & $\begin{array}{l}\text { Entrenamiento de un } \\
\text { deporte. }\end{array}$ & 2 & - & 2 & 4 \\
\hline & $\begin{array}{l}\text { Entrenamiento para } \\
\text { competencia. }\end{array}$ & $\mathbf{1}$ & - & 2 & 3 \\
\hline \multirow{12}{*}{\begin{tabular}{l}
\multicolumn{3}{l}{ Enfoques curriculares en } \\
clases de Educación \\
Fisica
\end{tabular}} & Clases Inclusivas. & $\mathbf{1}$ & 3 & $\mathbf{1}$ & 5 \\
\hline & Desarrollo cognitivo. & $\mathbf{1}$ & 3 & 2 & 7 \\
\hline & Desarrollo social. & $\mathbf{1}$ & 3 & 1 & 5 \\
\hline & Desarrollo afectivo. & 2 & 3 & $\mathbf{1}$ & 6 \\
\hline & Clases lúdicas. & 1 & 3 & 1 & 5 \\
\hline & $\begin{array}{l}\text { Desarrollo técnico } \\
\text { táctico. }\end{array}$ & $\mathbf{1}$ & 3 & $\mathbf{1}$ & 5 \\
\hline & $\begin{array}{l}\text { No competencias } \\
\text { deportivas. }\end{array}$ & $\mathbf{1}$ & 3 & $\mathbf{1}$ & 5 \\
\hline & $\begin{array}{l}\text { Desarrollo de } \begin{array}{r}\text { las } \\
\text { capacidades } \\
\text { adecuadas a la edad. }\end{array} \\
\text { adecuadas }\end{array}$ & $\mathbf{1}$ & 3 & 2 & 6 \\
\hline & $\begin{array}{ll}\text { Aplicación } & \text { de } \\
\text { metodologías activas de } \\
\text { enseñanza. }\end{array}$ & $\mathbf{1}$ & 3 & $\mathbf{1}$ & 5 \\
\hline & $\begin{array}{l}\text { Heteroevaluación, } \\
\text { coevaluación } \\
\text { autoevaluación. }\end{array}$ & 1 & 3 & $\mathbf{1}$ & 5 \\
\hline & Interacciones positivas. & 2 & 3 & 2 & 7 \\
\hline & Modelo constructivista. & $\mathbf{1}$ & 3 & $\mathbf{1}$ & 5 \\
\hline
\end{tabular}

Con base a los hallazgos se realizó una triangulación de datos espacial como se refieren Cohen y Manion (2002) al decir que la triangulación espacial se aplica cuando se concuerda entre las fuentes; también Rodríguez-Sabiote, Pozo-Llorente y Gutiérrez-Pérez (2006) 
mencionan que "se produce triangulación cuando las orientaciones cuantitativa y cualitativa se utilizan en el reconocimiento de un mismo e idéntico aspecto de la realidad social implementándose los métodos de forma independiente, pero orientados hacia una puesta en común", por otro lado Denzin (1970) comenta que la "triangulación es un procedimiento heurístico orientado a documentar y contrastar información según diferentes puntos de vista; de ahí que se pueda hablar de diferentes tipos de triangulación según el foco de contraste: técnicas, agentes, tiempos, métodos, o técnicas de análisis de datos"; es por eso que se triangulará las tres instituciones educativas cada una con criterios establecidos de acuerdo a las políticas de libertad curricular institucional.

A continuación se triangulará los datos de las entrevistas a docentes de tres instituciones educativas, agrupadas en dos criterios e interpretando cada una de las categorías.

\section{Criterio Entrenamiento Deportivo en las clases de Educación Física}

En este criterio se pudo agrupar todos los datos relacionados a la reflexión de los nueve docentes de Educación Física pertenecientes a tres instituciones educativas en referencia a varias categorías. Con los datos obtenidos se interpreta que los docentes conocen que el entrenamiento deportivo no es propio de la Educación Física, debido a que influyen factores pedagógicos y metodológicos propios de la educación; por lo que se entiende que el entrenamiento deportivo es excluyente, debido a que en las clases de Educación Física no todos los estudiantes de un grado tienen las cualidades y características para entrenar un determinado deporte, en consecuencia muchos no podrían participar de los entrenamientos quedando fuera de la clase, atentando con los principios educativos y la misión docente; de este modo queda descartado el perfeccionamiento de las técnicas y tácticas deportivas, porque la naturaleza de la Educación Física va enfocada al desarrollo de estas para la vida; y, si es para la vida no es necesario potencializar las capacidades físicas sino solo desarrollarlas, tomando en cuenta que si existen estudiantes con cualidades deportivas, se les motive a entrenamientos con profesionales especialistas en horario extracurricular.

De la misma forma en las clases de Educación Física no se puede hacer una detección de talentos deportivos, debido a que la mayoría de docentes no son especializados en esa área, lo que sí se puede es que si el estudiante tiene condiciones físicas, técnicas o tácticas orientarles para que entrene con un profesional especializado, la intención de las clases no es formar deportistas sino desarrollar estas habilidades deportivas que les sirva como estímulo y 
orientación para que tomen su decisión deportiva, por consiguiente en ningún caso se puede especializarlos en horario de Educación Física. Pero si la intención es competir se puede trabajar con la organización de festivales deportivos o campeonatos internos, proyectos que no se desarrollan en horario de Educación Física sino requieren de espacio y tiempos establecidos por las autoridades de la institución.

\section{Criterio Enfoques curriculares en clases de Educación Física}

Al igual que el criterio anterior agrupa los datos relacionados a la reflexión los docentes, pero sobre la visibilización de los enfoques curriculares en clases de Educación Física. De acuerdo a los enfoques curriculares dispuesto por el ajuste curricular las clases deben ser inclusivas, es decir todos los estudiantes participan de ella de forma activa y motivada, sin importar que el estudiante tenga alguna necesidad educativa asociada o no a la discapacidad, o simplemente no tenga las condiciones físicas o habilidades para realizar un determinado ejercicio, en ese sentido la construcción de las actividades sería la opción de aplicar una metodologías que adapte y genere la inclusión vinculando a que sea divertida y cause placer a todos los involucrados; en ese caso los estudiantes al construir cualquier actividad de acuerdo a sus posibilidades físicas aportarán en dicha construcción, pero en una construcción de actividades no solo el aporte físico es lo único, sino que existe el aporte intelectual, en que los estudiantes aportan con la búsqueda de soluciones y la elección de las mejores alternativas, un aporte social en la que los estudiantes se distribuirán responsabilidades y llegarán a acuerdos; y, un aporte afectivo en que la motivación y el ambiente de alegría con valores sea parte de las actividades; de esta forma la participación activa se garantiza en la construcción de la actividad.

En este mismo sentido en Educación Física se desarrolla las técnicas deportivas no solo de un deporte en especial, sino de varios deportes para que exista la posibilidad de que los estudiantes puedan orientarse por una disciplina; con esto la competencia deportiva en las clases de Educación Física no procede, porque las clases se deben ocupar para la enseñanza de la totalidad de los estudiantes y no solo para los que compiten que sería un grupo reducido, la naturaleza del entrenamiento deportivo no debe ser confundido con la naturaleza de la educación que es formar ciudadanos que se desarrollen de forma activa, aportando en la construcción de a misma, en cambio en entrenamiento aporta al mejoramiento de las capacidades físicas, perfeccionamiento de la técnica y táctica para obtener un rendimiento deportivo. 
Al igual que las otras asignaturas la Educación Física se alinea al modelo educativo Constructivista y con el se aplica las metodologías activas, como el aprendizaje cooperativo, aprendizaje basado en proyectos, aprendizaje basado en problemas, entre otros, garantizando el aprendizaje establecido en las destrezas con criterio de desempeño del bloque curricular Prácticas Deportivas, y su proceso de evaluación permanente, anclando las interacciones positivas en cada acción docente estudiante.

\section{REFLEXIONES FINALES}

Desde este marco de referencia investigativa, se debe manifestar el valor que se le concede a la participación activa, comprometida y desinteresada de los actores sociales, quienes al otorgarles la palabra, sin reserva alguna; narraban sus realidades, sentires, concepciones, haceres, acciones y reflexiones sobre el desarrollo del deporte y los enfoques curriculares en las clases de Educación Física, informaciones sustantivas sobre el fenómeno social en estudio, permitió la oportunidad de hacer de su bagaje experiencial y de conocimientos, así como de ciertas observaciones particulares que sobre la temática, que vivenciaban desde su contexto educativo.

Se considera también, como significativo para el quehacer investigativo en este contexto particular, haber adquirido la capacidad cognitiva y creativa para con confianza y credibilidad; comprender la naturaleza profunda de la realidad estudiada, que versaron los actores sociales con respecto a su estructura dinámica, aquella que dio razón plena de su comportamiento y manifestaciones, desde los cuales inductivamente se desprendieron hallazgos relevantes con ciertas condiciones, cualidades y características bien definidas, que permitieron interpretar los resultados encontrados.

En este orden de ideas, el significado y significante que asignan los docentes de Educación Física a la práctica del deporte en sus clases; es lo que consideran un fenómeno social arraigado, debido a que mezcla los currículos y los paradigmas educativos anteriores con el vigente, confundiendo notablemente la aplicabilidad de estos, obteniendo resultados no adecuados a los perfiles de salida del bachiller ecuatoriano, generando un impulso a nuevas investigaciones de experiencias educativas, que ayudarán con aportes que se alineen al modelo educativo, sostenida en la activación de actitudes, aptitudes, expectativas y motivaciones favorables para la práctica deportiva no como entrenamiento deportivo con fines de competir, 
sino de desarrollo de capacidades físicas, técnicas y tácticas deportivas de afines al desarrollo evolutivo de los estudiantes con fines de su desarrollo de la corporeidad y de adquirir una cultura de actividad física, que beneficie en esencia la formación integral del estudiante.

Los docentes fundamentan su accionar con comportamientos y acciones sustentados en la confianza, la credibilidad y la ejemplaridad. Asimismo, asumen su labor desde la premisa global de atención al estudiante en todas sus dimensiones, observando las necesidades específicas de cada uno de los estudiantes, convirtiéndose en un guía para que el estudiante construya las actividades en post del cumplimiento de los contenidos curriculares.

En este sentido son capaces de dirigir el talento deportivo observado, a especialistas deportivos que lo perfeccionarán extracurricularmente; quedándose con la misión educativa de nutrir las habilidades motrices, cognitivas, sociales y afectivas de cada contenido curricular, en función de lograr propiciar el desarrollo de la formación integral de sus estudiantes, lo que se constituye en el pilar fundamental en estos tiempos que ameritan sensibilizar al ser humano con respecto a sus actuaciones.

\section{REFERENCIAS}

Alonso, L. (2007). Sujetos y Discurso: el lugar de la entrevista abierta en las prácticas de la sociología cualitativa. Madrid, España: Editorial Síntesis.

American Psychological Association. (2010). Publication manual of the American Psychological Association. Washington: American Psychological Association.

Arufe, V. (2020). ¿Cómo debe ser el trabajo de Educación Física en Educación Infantil?. Retos 37, 588-596. Recuperado de https://recyt.fecyt.es/index.php/retos/article/view/74177/45764

Bernáez (2007). El deporte es concebido como una actividad de trabajo físico caracterizado por el espíritu de competencia reglamentada. Barcelona España: Paidos.

Cargua, A., Posso, R., Cargua, N. y Rodríguez, A. (2019). La formación del profesorado en el proceso de innovación y cambio educativo. OLIMPIA. Revista de la Facultad de Cultura Física de la Universidad de Granma, 16(54), 140-152.

Cohen, L. y Manion, L (2002). Métodos de investigación educativa. Madrid: La Muralla.

Denzin, N. K. (1970). The research act. Chicago: Aldine Publishing. 
Díaz-Bravo, L., Torruco-García, U., Martínez-Hernández, M. y Varela-Ruiz, M. (2013). La entrevista, recurso flexible y dinámico. Investigación en educación médica, 2(7), 162167. Recuperado de http://www.scielo.org.mx/scielo.php?script=sci_arttext\&pid=S2007$50572013000300009 \& \operatorname{lng}=$ es \&tlng=es.

Fernández, L. (2005) Bases metodológicas de la Iniciación y Especialización deportiva. Apuntes Congreso internacional de educación Física y Deporte .Cuernavaca, México.

Flores, G., Prat, M. y Soler, S. (2015). La intervención pedagógica del profesorado de educación física en un contexto multicultural: prácticas, reflexiones y orientaciones. Retos. Nuevas Tendencias en Educación Física, Deporte y Recreación, (28), 248-255.

Gómez, V., Padial, R., Gentil, M., Chacón, J. y Zurita, F. (2019). Implicaciones del Deporte Federado hacia la Empatía y Actitud a la Educación Física en Adolescentes. Retos, 36, 412-417. Recuperado de https://digibug.ugr.es/bitstream/handle/10481/59394/71582230443-1-PB.pdf? sequence $=1$ \&isAllowed=y

González-Martínez, L. (1993). Un acercamiento metodológico a la investigación cualitativa. Sinéctica, Revista Electrónica de Educación, (3), 1-12. Recuperado de https://www.redalyc.org/articulo.oa?id=998/99825983002

Hurtado, I. y Toro, G. (2001). Paradigmas y Métodos de Investigación en Tiempos de Cambio. Valencia: Episteme.

Labarrere, A. (1982). 'Las capacidades'. En Josefina López Hurtado. Psicología General. La Habana: Editorial Pueblo y Educación.

Maykut, P. y Morehouse, R. (1994). Beginning qualitative research a philosophical and practical guide. Londres: The Falmer Press.

Medranda-Rojas, J., Castillejo-Olán, R., Pérez-Ramírez, R. y Alonso-Betancourt, L. (2017). Fundamentos teóricos y metodológicos para la Identificación de talentos deportivos en la educación física Escolar. Revista Didascalía: Didáctica y Educación, 8(1), 1-18. Referenciado de http://refcale.uleam.edu.ec/index.php/didascalia/article/view/1661/913

Monzonís, N. y Capllonch, M. (2015). Mejorar la competencia social y ciudadana: innovación desde educación física y tutoría. RETOS. Nuevas Tendencias en Educación Física, Deporte $\quad y \quad$ Recreación, (28), 256-262.Recuperado de https://www.redalyc.org/articulo.oa?id=3457/345741428045

Organización de Naciones Unidas, UNESCO (1998), Conferencia intergubernamental sobre políticas culturales para el desarrollo. Estocolmo, Suecia, 30 de marzo - 2 de abril de 1998.

Platonov, V. N. (1987). Teoría del Deporte. Kiev: Ed. Escuela Superior.. 
Posso, R. (2018). Guía de estrategias metodológicas para Educación Física. Quito: Ministerio de Educación. Recuperado https:/educacion.gob.ec/wpcontent/uploads/downloads/2019/01/GUIA-METODOLOGICA-EF.pdf

Posso, R. Barba, L., Castro, R., Nuñez, F. y Marcillo, J. (2019). Enfoque lúdico como estrategia en el contexto de la Educación Física ecuatoriana: una revisión sistemática. Lecturas: Educación Física y Deportes, 24(258), 86-105. Recuperado de https://www.efdeportes.com/index.php/EFDeportes/article/view/1531/1021

Posso, R., Aponte, J., Zapata, M. y Betancourt, A. (2020). Aproximación fenomenológica y hermenéutica de los expertos en recreación, sobre las teorías del ocio y el tiempo libre en el proceso de socialización en las instituciones educativas, OLIMPIA. Revista de la Facultad de Cultura Física de la Universidad de Granma, 17(58), 78-91. Recuperado de https://revistas.udg.co.cu/index.php/olimpia/article/view/1269/2293

Posso, R., Barba, L., Marcillo, J., Acuña, M. y Hernández, F. (2020). Enfoques curriculares como contribución para la autonomía de la actividad física. EmásF. Revista Digital de Educación Física. (63), 132-145. Recuperado de https://emasf.webcindario.com/Enfoques_curriculares_como_contribucion_para_la_auto nomia_de_la_actividad_fisica.pdf

Posso, R., Otáñez, N., Guerrero, S., Betancourt, E. Noroña, L. y Manangón, R. (2020). Variables somatotípicas de nadadores y voleibolistas con proyección al alto rendimiento deportivo. Revista de Entrenamiento Deportivo. 34(1), 1-11. Recuperado de https://journal.onlineeducation.center/api-oas/v1/articles/sa-55e5936e4ab546/export-pdf

Renzi, G. (2009). Educación Física y su contribución al desarrollo integral de los niños en la primera infancia. Revista Iberoamericana de Educación, 7(50), 1-12. Recuperado de https://upvv.clavijero.edu.mx/cursos/LEB0739/documentos/EF_y_su_contribucion_al_de sarrollo_integral.pdf

Rodríguez-Sabiote, C., Pozo-Llorente, T. y Gutiérrez-Pérez, J. (2006). La triangulación analítica como recurso para la validación de estudios de encuesta recurrentes e investigaciones de réplica en Educación Superior. Relieve, 12(2), 289-305. http://www.uv.es/RELIEVE/v12n2/RELIEVEv12n2_6.htm.

Rodríguez-Rodríguez, F., Curilem-Gatica, C., Escobar-Gómez, D., y Valenzuela-Eberhard, L. (2016). Propuesta de evaluación de la educación física escolar en Chile. Educación Física y Ciencia, 18(1), 2-16.

Román, M. y Díez, E. (2000). El Currículum como Desarrollo de Procesos Cognitivos y Afectivos. Revista Enfoques Educacionales, 2(2), 1-20. Recuperado de https://semanariorepublicano.uchile.cl/index.php/REE/article/view/47064/49061

Skjong, R. y Wentworth, B. (2000). Expert Judgement and risk perception. Recuperado el 14 de Enero de 2020, de http://research.dnv.com/skj/Papers/SkjWen.pdf 
Soto, C.A. y Andújar, C. (2000) Reflexiones acerca del entrenamiento en la infancia y la selección de talentos deportivos. Lecturas: Educación Física y Deportes, 21, 1-7. Recuperado de https://www.efdeportes.com/efd21/talento.htm

Tinedo, M. (2012). Diseño de estrategias pedagógicas orientadas al estímulo de la práctica deportiva en los niños y niñas del centro educación inicial Carlos José Bello. (Tesis de Maestría) Universidad Latinoamericana del Caribe - ULAC, Estado Guárico Venezuela. 